\title{
Mutations in the Fas gene found in Spanish ALPS (autoimmune lymphoproliferative syndrome) patients
}

\author{
L Martínez-Martínez, C González-Santesteban, M V Rubiales, M Orta, I Badell, O de la Calle-Martin \\ From 5th European Workshop on Immune-Mediated Inflammatory Diseases \\ Sitges-Barcelona, Spain. 1-3 December 2010
}

\section{Background}

The autoimmune lymphoproliferative syndrome (ALPS) is a genetic disorder of lymphocyte apoptosis. ALPS is characterized by childhood onset of lymphadenopathy, hepatosplenomegaly, autoimmune cytopenias, elevated $(>1,5 \%)$ double negative T (DNT; CD3+TCR $\alpha \beta+C D 4-$ CD8-) lymphocytes in peripheral blood and an increased risk of lymphoma.

Most cases ( $>65 \%)$, known as ALPS type Ia, are associated with dominant heterozygous germline mutations in the TNFRSF6 gene encoding the protein for CD95 (Fas). Other genes are implicated in different ALPS subtypes (TNFSF6-Fas ligand, CASP8, CASP10 and N-RAS).

\section{Methods}

We studied 32 individuals from different Spanish Hospitals. Selected ALPS patients meet Clinical (lymphadenopathy, splenomegaly and/or cytopenias) and Laboratory (hyper-gammaglobulinemia and elevated DNT cells) criteria. TNFRSF6 gene was analyzed by DNA sequencing in every patient. In selected cases, without TNFRSF6 mutation, TNFSF6 (Fas ligand), CASP-8 (caspase-8), CASP-10 (caspase-10), N-RAS and FADD genes were further evaluated by sequencing.

\section{Results}

26 patients were found to be heterozygous for a single TNFRSF6 mutation (ALPS Ia).

Seven different alterations were identified affecting exons 2, 8 and 9. Four of these mutations have been not previously described (p.E20X, p.Y216fsX13, p.L226P and p.D210N). The mutations include insertions, splicing defects, missense and nonsense substitutions. The rest of the patients do not have any mutation in TNFRSF6, TNFSF6 or CASP-10 genes. Hence, they were classified as ALPS III. As compared to controls and healthy relatives bearing the same genetic alterations, ALPS Ia patients demonstrated significantly higher IL-10 and Igs serum levels, especially IgE. Those markers were also elevated in ALPS III patients but in a less remarkable fashion.

\section{Conclusions}

The combination of elevated DNT counts $(>1,5 \%)$ with increased IL-10 and IgE serum levels is strongly linked with clinical presentation in FAS mutation-bearing individuals, whereas isolated high DNT percentage is usually the only altered parameter in healthy carriers from the same families. This enable the targeting of patients with clinical features of ALPS to have a more directed evaluation, particularly in regard to DNA sequencing for FAS mutations and other apoptosis-related genes. Several novel FAS mutations were found and characterized in Spanish ALPS patients.

Published: 25 November 2010

doi:10.1186/1479-5876-8-S1-P20

Cite this article as: Martínez-Martínez et al:: Mutations in the Fas gene found in Spanish ALPS (autoimmune lymphoproliferative syndrome) patients. Journal of Translational Medicine 2010 8(Suppl 1):P20. 